\title{
Factores condicionantes de la adherencia al tratamiento con hierro en una cohorte de niños con anemia de $\mathbf{4}$ a $\mathbf{3 6}$ meses.
}

Conditioning factors for adherence to iron treatment in a cohort of children with anemia aged 4 to 36 months..

\section{RESUMEN}

Objetivo. Identificar los factores condicionantes de la adherencia al tratamiento con hierro en niños de 4 a 36 meses de edad, con anemia ferropénica.

Métodos. Se realizó un estudio observacional y transversal. La población muestral 97 niños de 4 a 36 meses de edad, usuarios de los centros de salud de Huánuco. Se les aplicaron un cuestionario de factores condicionantes de la adherencia al tratamiento con hierro, test de Morisky - Green y Levine, y la ficha de valoración del consumo de hierro y de anemia. Se aplicó la prueba chi cuadrado $y$ el odds ratio (OR) con un $p<0,05$.

Resultados. 91,8 \% (89) no se adhiere al tratamiento con hierro. Al analizar los factores institucionales, sociales, actitudinales y cognoscitivos condicionantes de la adherencia al tratamiento con hierro, solo hubo significancia en el factor institucional en su dimensión "el establecimiento de salud no otorga citas oportunas para la entrega del hierro" y en el factor actitudinal en sus dimensiones: "le toma demasiado tiempo hacer que el niño consuma el hierro", "olvido frecuente para darle el hierro al niño, deja de darle el tratamiento del hierro por que tuvo alguna experiencia negativa", "falta de tiempo para ir al establecimiento de salud a recoger el hierro", "suspensión del consumo de hierro por problemas respiratorios del niño". Todas éstas relacionadas con la no adherencia al tratamiento con hierro.

Conclusiones. El factor institucional y el factor actitudinal fueron los factores condicionantes de la no adherencia al tratamiento con hierro en los niños en estudio.

Palabras clave: Niño, anemia, Perú, factores condicionantes.

Universidad Nacional Mayor de San Marcos. Escuela Profesional de Medicina Humana. Lima-Perú.

Autor de Correspondencia: Mely Ruiz-Aquino (DD

E-mail: melyruizaquino@udh.edu.pe

Recibido: 17-03-2020

Aprobado: 03-07-2021

\begin{abstract}
Objective. The purpose of this study was to identify the conditioning factors for adherence to iron treatment in children between 4 and 36 months of age, with iron-deficiency anemia.
\end{abstract}

Methods. An observational and cross-sectional study was carried out. The sample population consisted of 97 children from 4 to 36 months of age, attending the health centers of Huánuco, Peru. A questionnaire of conditioning factors for adherence to iron treatment, the Morisky - Green and Levine test, and the assessment sheet for iron consumption and anemia were applied. The chi square test and the odds ratio $(O R)$ were used with a $p<0.05$.

Results. 91.8\% (89) did not adhere to iron treatment. When analyzing the institutional, social, attitudinal and cognitive factors conditioning adherence to iron treatment, there was only significance in the institutional factor in its dimension "the health facility does not give timely appointments for the delivery of iron" and in the attitudinal factor in its dimensions: "it takes too long for the child to consume iron", "frequent forgetting to give the child iron, stopped giving the iron treatment due to some negative experience", "lack of time to go to the health facility to collect iron "," interrupting iron consumption due to respiratory problems of the child ". All of these were factors related to the non-adherence to iron treatment.

Conclusions. The institutional factor and the attitudinal factor were the conditioning factors for non-adherence to iron treatment in the children under study.

Keywords: Child, anemia, Perú, conditioning factors.

\section{INTRODUCCIÓN}

La anemia es la anormalidad hematológica más común y prevalente en el mundo (1). Según la Organización Mundial de la Salud (OMS), la anemia infantil es considerada un problema de salud pública aún persistente. Se estima, además, que aproximadamente la mitad de casos de anemia se deben a la deficiencia de hierro. El hierro es el nutrimento inorgánico con más amplia distribución entre los niños, ya que participa en procesos vitales para el ser humano (2). Sin embargo, la anemia viene afectando alrededor de 800 millones de niños y mujeres, comprometiendo la habilidad del niño para aprender, limita aún más sus perspectivas de futuro; lo que, en términos agregados, dificulta el desarrollo de la población (3).

Al respecto, Marcacuzco (4), explica que la anemia por deficiencia de hierro en niños menores de cinco años ocupa uno de los primeros lugares en prevalencia e incidencia, a pesar de conocer su etiología y de las estrategias 
desarrolladas. Más aún, no se ha podido superar dicho problema de salud ya que se desconoce su impacto, nivel de adherencia o cumplimiento (5).

La suplementación preventiva se inicia con gotas a los 4 meses de vida (sulfato ferroso o complejo polimaltosado férrico en gotas), hasta cumplir los 6 meses de edad. Luego, se continúa con la entrega de micronutrientes desde los 6 meses de edad hasta completar 360 sobres de micronutrientes (1 sobre por día) (6).

Respecto a la magnitud del problema, la encuesta demográfica y de Salud (ENDES) del Instituto Nacional de Estadísticas e Informática (INEI) año 2015 reportó que $43,6 \%$ de los niños del Perú de entre 6 y 35 meses de edad, tuvo anemia; asimismo, la anemia afectó $60,7 \%$ entre los seis a ocho meses de edad y $63,1 \%$ de 9 a 11 meses de edad, siendo aún elevada en niños de 12 a 17 meses de edad (63\%) y de 18 a 23 meses de edad (47,9\%). La región más afectada es La Sierra, donde $51,8 \%$ de niños menores de 3 años tuvo anemia, siguiendo muy de cerca la zona selva (51,7\%). La Costa, por su parte, presenta 36,7\% (7).

Frente a ello, el Ministerio de Salud (MINSA) del Perú, a través de las Direcciones Regionales de Salud y las Unidades Ejecutoras (en el marco de sus competencias) vienen desarrollando diversas estrategias para prevenir y controlar la anemia ferropénica, priorizando a los niños de 6 a 36 meses (6), con la suplementación obligatoria con hierro (5), en los establecimientos de salud, como parte de la atención integral del niño en los consultorios de Control de Crecimiento y Desarrollo (CRED). Existen múltiples factores condicionantes a la adherencia al tratamiento con hierro como son los factores institucionales, actitudinales, cognitivos, sociales, entre otros (8-10).

Así, la temática de la anemia ferropénica en el infante es un problema prevalente e incidente en la actualidad; los factores condicionantes de la adherencia del tratamiento con hierro. Por ello, se busca proporcionar las evidencias científicas para desarrollar acciones de promoción de la salud y prevención de la enfermedad según factores condicionantes, siendo por tal el objetivo identificar los factores condicionantes de la adherencia al tratamiento con hierro en niños con anemia ferropénica de 4 a 36 meses de edad.

\section{MATERIALES Y MÉTODOS}

Estudio con enfoque cuantitativo, de tipo transversal y observacional, desarrollado en el año 2020. Se consideró a 97 niños de 4 a 36 meses de edad, usuarios de los centros de salud: Aparicio Pomares, Las Moras, Potracancha y La Esperanza, en la Provincia de Huánuco, en Perú. Se incluyeron a los niños con diagnóstico de anemia ferropénica durante el 2020, cuyas madres firmaron el consentimiento informado. Asimismo, se excluyeron a niños cuyas madres no consintieron su participación.

Instrumentos de recolección de datos. Se empleó una ficha de las características del consumo del hierro y una ficha clínica del análisis de hemoglobina, definiéndose anemia ferropénica cuando se encontraban valores menores de 110 $\mathrm{g} / \mathrm{L}$ de hemoglobina ajustada por altitud. También se usó el cuestionario de factores condicionantes de la adherencia al tratamiento con hierro, con 4 dimensiones: factor institucional (7 ítems), social (7 ítems), actitudinal (11 ítems) y cognoscitivo (5 ítems), llegando a un total de 30 ítems. También se aplicó el test de Morisky-Green y Levine para medir la adherencia al tratamiento de la anemia ferropénica, como método indirecto subjetivo, considerando adherente a aquel niño que cumplió con $75 \%$ de su tratamiento con hierro.

Los instrumentos de medición fueron validados por siete expertos en la temática del estudio. También se hizo un pilotaje previo para determinar la confiabilidad del cuestionario de factores condicionantes de la adherencia al tratamiento con hierro mediante el método $\mathrm{KR}$, en el cual se obtuvo un valor de 0,81 .

Se midieron otras variables sociodemográficas como la edad y género del niño en meses, la edad en años, la escolaridad de la madre, así como las características del consumo del hierro.

Para la recolección de datos se hicieron visitas domiciliarias a cada una de las madres de los niños de 4 a 36 meses de edad.

Análisis de datos. El tratamiento estadístico de los datos se realizó mediante el análisis porcentual de las frecuencias de respuesta a los ítems. En el análisis inferencial se aplicó la prueba de chi cuadrado y con un nivel de significación estadística de $p<0,05$. Para la determinación de los factores asociados se aplicó en el análisis mediante el odds ratio (OR) con sus intervalos de confianza al $95 \%$.

El estudio fue revisado y aprobado por el Comité Institucional de Ética en Investigación de la Universidad de Huánuco, Huánuco. Se aplicó el consentimiento informado y los datos fueron tratados con total confidencialidad

\section{RESULTADOS}

Respecto a las características demográficas de los niños en estudio, una importante proporción eran mayores de 24 meses [79,4\% (77)]; mientras que, alrededor de la mitad eran del género masculino [52,6\% (51)]. Con respecto a las características alimentarias, se evidenció que 92,8\% (90) cumplieron con la lactancia materna exclusiva, mientras que $84,5 \%$ (82) la lactancia fue a libre demanda. El 49,5\% (48) iniciaron su alimentación complementaria luego de los 7 meses de edad y 79,4\% (77) consumían cinco comidas al día.

Analizando las características del consumo de hierro, 62,9\% (61) de los niños consumieron el hierro polimaltosado, dosificado con una cuchara; en 95,9\% (93), el consumo de hierro fue administrado de forma diaria (Tabla 1). En el 43,3\% (42) acompañaron el consumo de hierro con un alimento sólido como las papillas. Con respecto a los efectos adversos, una importante proporción [26,8\% (26)] percibieron diarrea. 
Tabla 1. Características del consumo del hierro de los niños usuarios de los establecimientos de salud de Huánuco.

\begin{tabular}{|l|c|c|}
\hline Características del consumo de hierro & \multicolumn{2}{|c|}{$\mathbf{n = 9 7}$} \\
\hline Tipo de hierro & f & $\%$ \\
\hline Sulfato ferroso & 36 & 37,1 \\
\hline Hierro polimaltosado & 61 & 62,9 \\
\hline Forma farmacéutica & & \\
\hline Solución & 36 & 37,1 \\
\hline Jarabe & 61 & 62,9 \\
\hline Dosis & & \\
\hline Gotas & 61 & 62,9 \\
\hline Cucharadas & 36 & 37,1 \\
\hline Frecuencia & & \\
\hline Diaria & 93 & 95,9 \\
\hline Interdiaria & 4 & 4,1 \\
\hline Tiempo de consumo & & \\
\hline Menos de 5 minutos & 68 & 70,1 \\
\hline De 6 a 10 minutos & 20 & 20,6 \\
\hline De 11 a 15 minutos & 7 & 7,2 \\
\hline Más de 15 minutos & 2 & 2,1 \\
\hline
\end{tabular}

Respecto al nivel de hemoglobina de los niños en estudio, se halló que, antes del consumo de hierro, una importante proporción tuvieron anemia leve $[59,8 \%$ (58)]; mientras que, después del consumo de hierro, mostraron un nivel de hemoglobina normal $(71,1 \%)$ (Fig. 1).

$\begin{array}{llll}0,0 & 10,0 \quad 20,0 \quad 30,0 \quad 40,0 \quad 50,0 \quad 60,0 \quad 70,0 \quad 80,0\end{array}$

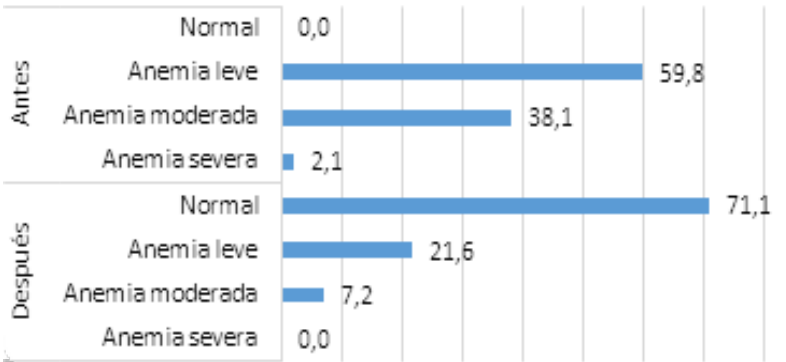

Fig. 1. Representación de los valores de hemoglobina en los niños, antes y después del consumo de hierro.

En la adherencia al tratamiento con hierro en niños con anemia ferropénica de 4 a 36 meses en estudio, se evidenció que 57,7\% (56) "le brinda el hierro en el horario indicado"; 45,4\% (44) "ha dejado de dar el tratamiento del hierro cuando el niño bajo su cuidado enferma de diarrea o tos"; $32 \%$ (31) "ha olvidado alguna vez dar el tratamiento de hierro a su niño"; y 23,7\% (23) "cuándo el niño se siente bien, está alegre, animado y se ve sano, deja de darle el tratamiento del hierro". Al analizar de forma conjunta la adherencia al tratamiento con hierro en niños con anemia ferropénica de 4 a 36 meses en estudio, se halló 98\% (89) "no se adhiere al tratamiento con hierro" frente $8.2 \%$ (8) que si presentó adherencia (Tabla 2).

Tabla 2. Adherencia al tratamiento.

\begin{tabular}{|l|c|c|c|c|c|}
\hline & \multicolumn{4}{c|}{ n = 97 } \\
\hline Adherencia al tratamiento & \multicolumn{2}{|c|}{ Sí } & \multicolumn{2}{|c|}{ No } \\
\hline \begin{tabular}{l|l|l|l|}
\hline Ha olvidado alguna vez dar el \\
tratamiento de hierro a su niño.
\end{tabular} & 31 & 32,0 & 66 & 68,0 \\
\hline $\begin{array}{l}\text { Cuando su niño se siente bien, está } \\
\text { alegre, animado y se ve sano deja } \\
\text { de darle el tratamiento del hierro. }\end{array}$ & 23 & 23,7 & 74 & 76,3 \\
\hline $\begin{array}{l}\text { Ha dejado de dar el tratamiento } \\
\text { del hierro cuando el niño bajo su }\end{array}$ & 44 & 45,4 & 53 & 54,6 \\
\hline $\begin{array}{l}\text { cuidado se enferma, por ejemplo, de } \\
\text { diarrea o tos. }\end{array}$ & & & & \\
\hline $\begin{array}{l}\text { Le brinda el tratamiento del hierro, } \\
\text { en el horario indicado. }\end{array}$ & 56 & 57,7 & 41 & 42,3 \\
\hline Total & 8 & 8,2 & 89 & 91,8 \\
\hline
\end{tabular}

Test de Morisky - Green y Levine.

A nivel inferencial, los factores institucionales condicionantes de la adherencia al tratamiento con hierro en niños con anemia ferropénica de 4 a 36 meses, solo "hecho de que el establecimiento de salud no otorga citas oportunas para la entrega del hierro" [(x2 = 6,972; $p$-valor $=0,008)]$ tuvo relación con la adherencia al tratamiento. Al evaluar la probabilidad de riesgo, este representó hasta 6,9 veces más de riesgo para la no adherencia al tratamiento (Tabla 3).

Al comparar los factores sociales condicionantes de la adherencia al tratamiento con hierro en niños con anemia de 4 a 36 meses, ningún factor social resultó ser significativo (Tabla 4).

Al confrontar los factores actitudinales condicionantes de la adherencia al tratamiento con hierro en niños con anemia ferropénica, fueron significativos: "le toma demasiado tiempo hacer que el niño consuma el hierro" [( $x 2=5,041$; $p$-valor = $0,025)]$, "olvido frecuente para darle el hierro al niño" [(x2 = $15,452$; p-valor $=0,000)$ ], "deja de darle el tratamiento del hierro porque tuvo alguna experiencia negativa" $[(x 2=7,884$; $\mathrm{p}$-valor $=0,014)]$, "Falta de tiempo para ir al establecimiento de salud a recoger el hierro" $[(x 2=9,484 ; p-$ valor $=$ 0,002)], "Suspensión del consumo de hierro por problemas respiratorios del niño" [(x2 = 7,310; $p$ - valor $=0,007)]$; todas estas fueron condicionantes de la adherencia al tratamiento con hierro. Al evaluar la probabilidad de riesgo, "el olvido frecuente para darle el hierro al niño" fue 24 veces más de riesgo para la no adherencia al tratamiento con hierro (Tabla 5).

Evaluando los factores cognoscitivos condicionantes de la adherencia al tratamiento con hierro en niños con anemia ferropénica de 4 a 36 meses, se comprobó que ninguno de ellos resultó ser significativo (Tabla 6). 
Tabla 3. Factores institucionales condicionantes de la adherencia al tratamiento con hierro en niños con anemia de 4 a 36 meses.

\begin{tabular}{|c|c|c|c|c|c|c|c|c|c|}
\hline \multirow{3}{*}{ Factores institucionales } & & \multicolumn{4}{|c|}{ Adherencia al tratamiento con hierro } & \multirow[t]{3}{*}{$\mathbf{X} 2$} & \multirow[t]{3}{*}{ OR } & \multirow[t]{3}{*}{ IC } & \multirow[t]{3}{*}{ p-valor } \\
\hline & & \multicolumn{2}{|c|}{ Sí } & \multicolumn{2}{|c|}{ No } & & & & \\
\hline & & $f$ & $\%$ & $f$ & $\%$ & & & & \\
\hline \multirow{2}{*}{$\begin{array}{l}\text { El establecimiento de salud le brinda } \\
\text { el tratamiento de la anemia de manera } \\
\text { oportuna }\end{array}$} & Sí & 8 & 8,2 & 85 & 87,6 & 0,375 & 1,094 & $(1,03-1,17)$ & 0,540 \\
\hline & No & 0 & 0,0 & 4 & 4,1 & & & & \\
\hline \multirow{2}{*}{$\begin{array}{l}\text { Recibió la consejería nutricional por el } \\
\text { personal de salud }\end{array}$} & Sí & 7 & 7,2 & 72 & 74,2 & 0,212 & 1,653 & $(0,19-14,35)$ & 0,645 \\
\hline & No & 1 & 1,0 & 17 & 17,5 & & & & \\
\hline \multirow{2}{*}{$\begin{array}{l}\text { Recibió visitas domiciliarias del personal de } \\
\text { salud, para orientar el consumo del hierro }\end{array}$} & Sí & 6 & 6,2 & 43 & 44,3 & 2,091 & 3,209 & $(0,61-16,77)$ & 0,148 \\
\hline & No & 2 & 2,1 & 46 & 47,4 & & & & \\
\hline \multirow{2}{*}{$\begin{array}{l}\text { El personal de salud le enseñó a administrar } \\
\text { el hierro }\end{array}$} & Sí & 7 & 7,2 & 85 & 87,6 & 0,962 & 0,329 & $(0,03-3,36)$ & 0,327 \\
\hline & No & 1 & 1,0 & 4 & 4,1 & & & & \\
\hline \multirow{2}{*}{$\begin{array}{l}\text { El personal de salud le explicó sobre los } \\
\text { efectos secundarios del hierro }\end{array}$} & Sí & 7 & 7,2 & 70 & 72,2 & 0,351 & 1,900 & $(0,22-16,41)$ & 0,553 \\
\hline & No & 1 & 1,0 & 19 & 19,6 & & & & \\
\hline \multirow{2}{*}{$\begin{array}{l}\text { Recibió información clara o entendible sobre } \\
\text { el tratamiento con hierro para prevenir la } \\
\text { anemia }\end{array}$} & Sí & 7 & 7,2 & 78 & 80,4 & 0,000 & 0,987 & $(0,11-8,81)$ & 0,667 \\
\hline & No & 1 & 1,0 & 11 & 11,3 & & & & \\
\hline \multirow{2}{*}{$\begin{array}{l}\text { El establecimiento de salud le da las citas } \\
\text { oportunas para la entrega del hierro }\end{array}$} & Sí & 5 & 5,2 & 82 & 84,5 & 6,972 & 0,142 & $(0,03-0,72)$ & 0,008 \\
\hline & No & 3 & 3,1 & 7 & 7,2 & & & & \\
\hline Total & & 8 & 8,2 & 89 & 91,8 & & & & \\
\hline
\end{tabular}

Tabla 4. Factores sociales condicionantes de la adherencia al tratamiento con hierro en niños con anemia de 4 a 36 meses.

\begin{tabular}{|c|c|c|c|c|c|c|c|c|c|}
\hline \multirow[t]{3}{*}{ Factores sociales } & & \multicolumn{4}{|c|}{$\begin{array}{c}\text { Adherencia al tratamiento con } \\
\text { hierro }\end{array}$} & \multirow[t]{3}{*}{$\mathrm{X} 2$} & \multirow[t]{3}{*}{ OR } & \multirow[t]{3}{*}{ IC } & \multirow[t]{3}{*}{ p-valor } \\
\hline & & \multicolumn{2}{|c|}{ Sí } & \multicolumn{2}{|c|}{ No } & & & & \\
\hline & & $f$ & $\%$ & $f$ & $\%$ & & & & \\
\hline \multirow{2}{*}{$\begin{array}{l}\text { En casa le apoyan con el consumo del } \\
\text { hierro }\end{array}$} & Sí & 7 & 7,2 & 78 & 80,4 & 0,00 & 0,98 & $(0,11-8,81)$ & 0,991 \\
\hline & No & 1 & 1,0 & 11 & 11,3 & & & & \\
\hline \multirow{2}{*}{$\begin{array}{l}\text { Su familia incentiva que el niño } \\
\text { consuma el hierro }\end{array}$} & Sí & 7 & 7,2 & 83 & 85,6 & 0,364 & 0,506 & $(0,05-4,82)$ & 0,547 \\
\hline & No & 1 & 1,0 & 6 & 6,2 & & & & \\
\hline \multirow{2}{*}{$\begin{array}{l}\text { Los vecinos de su comunidad, } \\
\text { tienen comentarios positivos sobre el } \\
\text { consumo de hierro }\end{array}$} & Sí & 7 & 7,2 & 52 & 53,6 & 2,604 & 4,981 & $(0,59-2,22)$ & 0,107 \\
\hline & No & 1 & 1,0 & 37 & 38,1 & & & & \\
\hline \multirow{2}{*}{$\begin{array}{l}\text { En casa algún familiar le recuerda que } \\
\text { debe dar hierro a su niño }\end{array}$} & Sí & 7 & 7,2 & 73 & 75,3 & 0,152 & 1,534 & $(0,18-3,36)$ & 0,696 \\
\hline & No & 1 & 1,0 & 16 & 16,5 & & & & \\
\hline \multirow{2}{*}{$\begin{array}{l}\text { Las veces que no pudo darle el hierro } \\
\text { a su niño, le deja encargado a algún } \\
\text { familiar }\end{array}$} & Sí & 7 & 7,2 & 64 & 66,0 & 0,909 & 2,734 & $(0,32-3,37)$ & 0,340 \\
\hline & No & 1 & 1,0 & 25 & 25,8 & & & & \\
\hline \multirow{2}{*}{$\begin{array}{l}\text { Recibe apoyo familiar en la crianza o } \\
\text { cuidado de su niño }\end{array}$} & Sí & 6 & 6,2 & 68 & 70,1 & 0,008 & 0,926 & $(0,17-4,94)$ & 0,929 \\
\hline & No & 2 & 2,1 & 21 & 21,6 & & & & \\
\hline \multirow{2}{*}{$\begin{array}{l}\text { Le parece importante que el personal } \\
\text { de salud le visite su casa para } \\
\text { preguntarle sobre el consumo del } \\
\text { hierro }\end{array}$} & Sí & 8 & 8,2 & 80 & 82,5 & 0,892 & 1,100 & $(1,03-1,18)$ & 0,345 \\
\hline & No & 0 & 0,0 & 9 & 9,3 & & & & \\
\hline Total & & 8 & 8,2 & 89 & 91,8 & & & & \\
\hline
\end{tabular}


Tabla 5. Factores actitudinales condicionantes de la no adherencia al tratamiento con hierro en niños con anemia de 4 a 36 meses.

\begin{tabular}{|c|c|c|c|c|c|c|c|c|c|}
\hline \multirow[t]{3}{*}{ Factores actitudinales } & & \multicolumn{4}{|c|}{$\begin{array}{l}\text { Adherencia al } \\
\text { tratamiento con hierro }\end{array}$} & \multirow[t]{3}{*}{$\mathbf{X} 2$} & \multirow[t]{3}{*}{ OR } & \multirow[t]{3}{*}{ IC } & \multirow[t]{3}{*}{ p-valor } \\
\hline & & \multicolumn{2}{|c|}{ Sí } & \multicolumn{2}{|c|}{ No } & & & & \\
\hline & & $f$ & $\%$ & $f$ & $\%$ & & & & \\
\hline \multirow{2}{*}{$\begin{array}{l}\text { Considera que la anemia es un problema común de } \\
\text { salud }\end{array}$} & Sí & 8 & 8,2 & 86 & 88,7 & 0,278 & 1,093 & $(1,03-1,16)$ & 0,598 \\
\hline & No & 0 & 0,0 & 3 & 3,1 & & & & \\
\hline \multirow{2}{*}{$\begin{array}{l}\text { Considera que la anemia se cura solo con una } \\
\text { alimentación rica en hierro }\end{array}$} & Sí & 8 & 8,2 & 83 & 85,6 & 0,575 & 1,096 & $(1,03-1,17)$ & 0,448 \\
\hline & No & 0 & 0,0 & 6 & 6,2 & & & & \\
\hline \multirow{2}{*}{$\begin{array}{l}\text { Siente temor de que el hierro le pueda causar } \\
\text { estreñimiento, diarrea u otra molestia a su niño }\end{array}$} & Sí & 8 & 8,2 & 66 & 68,0 & 2,710 & 1,121 & $(1,04-1,21)$ & 0,100 \\
\hline & No & 0 & 0,0 & 23 & 23,7 & & & & \\
\hline \multirow{2}{*}{$\begin{array}{l}\text { Le toma demasiado tiempo hacer que su niño consuma } \\
\text { el hierro }\end{array}$} & Sí & 7 & 7,2 & 41 & 42,3 & 5,041 & 8,195 & $(0,97-9,40)$ & 0,025 \\
\hline & No & 1 & 1,0 & 48 & 49,5 & & & & \\
\hline \multirow{2}{*}{$\begin{array}{l}\text { Considera importante la consejería del personal de } \\
\text { salud sobre el tratamiento con hierro }\end{array}$} & Sí & 8 & 8,2 & 78 & 80,4 & 1,115 & 1,103 & $(1,03-1,18)$ & 0,291 \\
\hline & No & 0 & 0,0 & 11 & 11,3 & & & & \\
\hline \multirow{2}{*}{ Olvido frecuente para darle el hierro a su niño } & Sí & 7 & 7,2 & 20 & 20,6 & 15,452 & 24,15 & $(2,80-208,0)$ & 0,000 \\
\hline & No & 1 & 1,0 & 69 & 71,1 & & & & \\
\hline \multirow{2}{*}{$\begin{array}{l}\text { Percibe poca mejoría de la anemia aun estando } \\
\text { consumiendo el hierro }\end{array}$} & Sí & 8 & 8,2 & 77 & 79,4 & 1,231 & 1,104 & $(1,03-1,18)$ & 0,267 \\
\hline & No & 0 & 0,0 & 12 & 12,4 & & & & \\
\hline \multirow{2}{*}{$\begin{array}{l}\text { Deja de darle el tratamiento al niño del hierro porque } \\
\text { tuvo alguna experiencia negativa }\end{array}$} & Sí & 5 & 5,2 & 17 & 17,5 & 7,884 & 7,059 & $(1,54-32,46)$ & 0,014 \\
\hline & No & 3 & 3,1 & 72 & 74,2 & & & & \\
\hline \multirow{2}{*}{$\begin{array}{l}\text { Falta de tiempo para ir al establecimiento de salud a } \\
\text { recoger el hierro }\end{array}$} & Sí & 7 & 7,2 & 29 & 29,9 & 9,484 & 14,48 & $(1,70-123,3)$ & 0,002 \\
\hline & No & 1 & 1,0 & 60 & 61,9 & & & & \\
\hline \multirow{2}{*}{$\begin{array}{l}\text { Suspensión del consumo de hierro por problemas } \\
\text { respiratorios del niño }\end{array}$} & Sí & 7 & 7,2 & 34 & 35,1 & 7,310 & 11,32 & $(1,33-96,10)$ & 0,007 \\
\hline & No & 1 & 1,0 & 55 & 56,7 & & & & \\
\hline \multirow{2}{*}{ Le interesa curar la anemia de su niño } & Sí & 8 & 8,2 & 77 & 79,4 & 1,231 & 1,104 & $(1,03-1,18)$ & 0,267 \\
\hline & No & 0 & 0,0 & 12 & 12,4 & & & & \\
\hline Total & & 8 & 8,2 & 89 & 91,8 & & & & \\
\hline
\end{tabular}

Tabla 6. Factores cognoscitivos condicionantes de la adherencia al tratamiento con hierro en niños con anemia de 4 a 36 meses.

\begin{tabular}{|c|c|c|c|c|c|c|c|c|c|}
\hline \multirow[t]{3}{*}{ Factores cognoscitivos } & & \multicolumn{4}{|c|}{$\begin{array}{l}\text { Adherencia al tratamiento con } \\
\text { hierro }\end{array}$} & \multirow[t]{3}{*}{$\mathbf{X} 2$} & \multirow[t]{3}{*}{ OR } & \multirow[t]{3}{*}{ IC } & \multirow[t]{3}{*}{ p-valor } \\
\hline & & \multicolumn{2}{|c|}{ Sí } & \multicolumn{2}{|c|}{ No } & & & & \\
\hline & & $f$ & $\%$ & $f$ & $\%$ & & & & \\
\hline \multirow[t]{2}{*}{ Sabe qué es la anemia ferropénica } & Sí & 7 & 7,2 & 87 & 89,7 & 2,574 & 0,161 & $(0,01-2,00)$ & 0,109 \\
\hline & No & 1 & 1,0 & 2 & 2,1 & & & & \\
\hline \multirow[t]{2}{*}{ Sabe cuándo su niño tiene anemia } & Sí & 7 & 7,2 & 84 & 86,6 & 0,599 & 0,417 & $(0,04-4,08)$ & 0,439 \\
\hline & No & 1 & 1,0 & 5 & 5,2 & & & & \\
\hline \multirow[t]{2}{*}{ Conoce los beneficios del hierro } & Sí & 7 & 7,2 & 87 & 89,7 & 2,574 & 0,161 & $(0,01-2,00)$ & 0,109 \\
\hline & No & 1 & 1,0 & 2 & 2,1 & & & & \\
\hline \multirow[t]{2}{*}{ Conoce los signos y síntomas de una anemia } & Sí & 7 & 7,2 & 87 & 89,7 & 2,574 & 0,161 & $(0,01-2,00)$ & 0,109 \\
\hline & No & 1 & 1,0 & 2 & 2,1 & & & & \\
\hline \multirow[t]{2}{*}{$\begin{array}{l}\text { Conoce los efectos secundarios que pueda } \\
\text { causar el hierro en su niño }\end{array}$} & Sí & 7 & 7,2 & 75 & 77,3 & 0,059 & 1,307 & $(0,15-1,46)$ & 0,809 \\
\hline & No & 1 & 1,0 & 14 & 14,4 & & & & \\
\hline \multirow[t]{2}{*}{$\begin{array}{l}\text { Conoce las complicaciones de la anemia no } \\
\text { tratada con hierro }\end{array}$} & Sí & 7 & 7,2 & 73 & 75,3 & 0,152 & 1,534 & $(0,18-13,36)$ & 0,696 \\
\hline & No & 1 & 1,0 & 16 & 16,5 & & & & \\
\hline Total & & 8 & 8,2 & 89 & 91,8 & & & & \\
\hline
\end{tabular}




\section{DISCUSIÓN}

Se evidenció que una alta proporción de la muestra no se adhiere al tratamiento con hierro. De forma similar, Munares y Gómez (5), hallaron una baja prevalencia de adherencia para un punto de corte exigente ( $\geq 90 \%$ sobres de multimicronutrientes consumidos). Castro (6), señala que las madres incumplen el tratamiento con hierro gotas hierro porque al niño no le gusta y además le genera efectos secundarios.

Según el reporte del Instituto de Nacional de Estadística e Informática del Perú (11), la adherencia promedio a la suplementación con hierro ha sido de $23,3 \%$ en los últimos cinco años (3), lo que explicaría el exiguo cambio que se ha visto en la tendencia de prevalencia de la anemia ferropénica en los últimos años, ya que ha permanecido constante con alrededor del $43 \%$ de casos. Tinoco et al. (12), encontraron que $53,3 \%$ de madres presentó una adherencia media, mientras que 53,3 \% de niños presentó hemoglobina normal. Además, de acuerdo al mismo estudio, no existe relación entre la adherencia general a la suplementación con multimicronutrientes y el nivel de hemoglobina.

Ortega et al. (13), menciona la falta de adherencia al tratamiento es la principal causa de que no se obtengan todos los beneficios que los medicamentos pueden proporcionar a los pacientes; esto constituye un hecho de fracaso terapéutico. Por su parte Ramos (14), explica que la adherencia al tratamiento resulta un proceso muy complejo que requiere aún de educación para la salud en la población y también a los prestadores de salud, además de la participación activa y responsable del mismo individuo, familiar y comunidad.

Resultados diferentes encontró Laura (15), quien indicó que $60 \%$ de las madres con lactantes de 4 meses tuvo adherencia a la suplementación con sulfato ferroso en gotas.

Echagüe et al. (16), realizaron una evaluación de la anemia ferropénica posintervención nutricional en niños de comunidades rurales de Caazapá, en Paraguay, encontraron que ambas poblaciones de niños anémicos indígenas y no indígenas presentaron un aumento significativo de los niveles medios de hemoglobina postratamiento con suplemento de hierro.

También se halló la dimensión del factor institucional "El establecimiento de salud no otorga citas oportunas para la entrega del hierro" es un factor condicionante de la no adherencia del tratamiento con hierro". Al respecto, Huamán et al. (17), explican que no basta con entregar o consumir la cantidad necesaria de los multimicronutrientes, o dar citas, sino asegurar que el proceso de consumo sea adecuado para lograr una reducción de la anemia; aspecto que debe ser trabajado para mejorar esta intervención.

Ramos (14), refiere que los servicios de salud poco desarrollados, la falta de conocimiento y adiestramiento del personal de salud en el control de las enfermedades o el personal con recargado horario de trabajo, influyen en la falta de adherencia de los tratamientos médicos.

Aparco y Huamán (18), encontraron que las barreras inherentes al sistema de salud se dividen, además, en dos grandes categorías: barreras para obtener una atención en el establecimiento de salud y barreras durante el proceso de recibir los multimicronutrientes. Las madres indican dificultades para acceder al establecimiento de salud, ya que el suplemento se recogía (o se obtenía la orden) en el consultorio de CRED, uno de los servicios con mayor demanda, que establece un número fijo de niños (cupo) para atención en el día.

Similares resultados reportaron Inca y Munares (19), indicando que existen factores relacionados a la prestación de servicios de salud que incrementan la adherencia al tratamiento hierro.

En un tercer momento se encontró que los factores actitudinales son las condicionantes de la adherencia del tratamiento con hierro. Al respecto, Chávez (20), acertó que los factores actitudinales como la confianza hacia los multimicronutrientes, el olvido y la percepción del beneficio, están asociadas a la falta de adherencia en el consumo de los multimicronutriente.

Vargas (21), halló que la mayoría de las madres de niños de 6 a 36 meses presentan actitudes desfavorables hacia la suplementación con los multimicronutrientes. En tanto Ipanaqué y Taype (22) hallaron que, en su mayoría, las madres presentan una actitud poco adecuada $(56,9 \%)$ e inadecuada con un (43,1\%); cabe resaltar que ninguna de las madres encuestadas presentó una actitud adecuada.

Por su parte, Aparco y Huamán (18), encontraron que, en las zonas urbanas, muchas madres refieren que no tienen tiempo para asistir al establecimiento de salud a recoger los multimicronutrientes, porque estudian o trabajan y consideran que el tiempo a invertir para el recojo es excesivo. Mientras que, en el ámbito rural, algunas madres no acuden al establecimiento de salud porque están en temporada de siembra o no tienen los recursos para movilizarse.

Apolinario et al. (23), hallaron que los factores institucionales relacionados a las prácticas inadecuadas fueron: distribución de los micronutrientes y educación; mientras que, en relación a las prácticas adecuadas fue: visita domiciliaria. Como limitaciones se reconoce, en particular, el sesgo de medición generado por el autoreporte de la madre/padre; asimismo, la muestra reducida de niños no permite que los resultados sean conclusivos y estos solo son validados para el contexto del estudio.

Conclusiones. Una alta proporción de la muestra no se adhiere al tratamiento con hierro. Al analizar los factores institucionales, sociales, actitudinales y cognoscitivos 
condicionante de la adherencia al tratamiento con hierro en niños con anemia. Solo se halló significancia en el factor institucional en su dimensión "el establecimiento de salud no otorga citas oportunas para la entrega del hierro" y el factor actitudinal en sus dimensiones: "le toma demasiado tiempo hacer que el niño consuma el hierro", "olvida frecuente para darle el hierro al niño", "deja de darle el tratamiento al niño del hierro porque tuvo alguna experiencia negativa", "falta de tiempo para ir al establecimiento de salud a recoger el hierro", "suspensión del consumo de hierro por problemas respiratorios del niño"; todas estas variables estuvieron, en definitiva, fueron las condicionantes de la no adherencia al tratamiento con hierro.

Recomendaciones. A tenor de nuestros hallazgos se recomienda fortalecer la adherencia al tratamiento con hierro con mayor educación inclusiva a la población y fortalecimiento de capacidades profesionales al personal de salud, para el inicio adecuado de la suplementación con hierro. Transformar las percepciones, expectativas y motivaciones de las madres o tutores de los niños en metas y objetivos alcanzables, a través de una comunicación asertiva, y la toma de decisiones de mutuo acuerdo para involucrar de manera más activa a los padres de niño con anemia en el autocuidado del mismo.

Agradecimientos. Los estudiantes del Programa Académico de Enfermería de la Universidad de Huánuco, Huánuco, por haber participado en el diseño del estudio; en especial a Sofía Isabel Martel Parra, Fresia Morales Canteño, Alexandra Medina Zambrano y a los demás estudiantes del IX semestre-2019. A la Licenciada Liz Pino Mejía, coordinadora, de la estrategia sanitaria Alimentación y Nutrición Saludable (ESANS) de la Red de Salud de Huánuco.

\section{REFERENCIAS BIBLIOGRÁFICAS}

1. Ministerio de Salud (MINSA). Norma Técnica - Manejo Terapéutico y Preventivo de la Anemia en Niños, Adolescentes, Mujeres Gestantes y Puérperas [internet] [consultado el 15 de mayo del 2017] Disponible en: http://bvs.minsa.gob.pe/local/ MINSA/4190.pdf

2. Tostado-Madrid T, Benítez-Ruiz I, Pinzón-Navarro A, BautistaSilva M, Ramírez-Mayans JA. Actualidades de las características del hierro y su uso en pediatría. Acta pediátrica de México [Internet] 2015; 36(3): 189-200 [Consultado 2021 Mar 24] Disponible en: http://www.scielo.org.mx/scielo.php?script=sci_ arttext\&pid=S0186-23912015000300008\&lng=es\&tlng=es

3. Organización Panamericana de Salud; Organización mundial de la salud. Oficina Regional para las América. Panorama de la seguridad alimentaria y nutricional sistemas alimentarios sostenibles para poner fin al hambre y la malnutrición. [Internet] [consultado 2018 Nov 2]. Disponible en: https://iris.paho.org/ handle/10665.2/34343

4. Marcacuzco A. Factores asociados a la adherencia de suplementación con micronutrientes en niños menores de 3 años de las Aldeas SOS, San Juan de Lurigancho-2017 [Internet] Lima: Universidad Cesar Vallejo; 2018 [consultado 2019 Oct 15]. Disponible en: https://revistas.unilibre.edu.co/ index.php/rcslibre/article/view/4980/4381
5. Munares O, Gómez G. Adherencia a multimicronutrientes y factores asociados en niños de 6 a 35 meses de sitios centinela, Ministerio de Salud, Perú. Rev Brasil Epidemiol. [Internet] 2016 Sep; 19(3): 539-553 [consultado 2021 Feb 09]. Disponible: http://www.scielo.br/scielo.php?script=sci_arttext\&pid=S141 5790X2016000300539\&Ing=en.https://doi.org/10.1590/19805497201600030006

6. Munares-García O, Gómez-Guizado G. Adherencia a multimicronutrientes y factores asociados en niños de 6 a 35 meses de sitios centinela, Ministerio de Salud, Perú. Revista Brasileira de Epidemiologia [Internet] 2016; 19(3): 539-553 [Consultado 2021 Mar 24] Disponible en: https://doi. org/10.1590/1980-5497201600030006

7. Instituto Nacional de Estadística e Informática. Encuesta demográfica y de Salud Familiar-ENDES [Internet] [consultado 2017 Mar 12] Disponible en: https://www.inei.gob.pe/media/ MenuRecursivo/publicaciones_digitales/Est/Lib1211/pdf/Libro. pdf-***

8. Santa Cruz L. Factores socioculturales que influyen en la aceptabilidad y consumo de los multimicronutrientes en las familias con niños menores de 3 años. Chilete-2015 [Internet] Cajamarca: Universidad Nacional de Cajamarca Escuela de Posgrado; 2017 [consultado 2019 Nov 15] Disponible en: http:// repositorio.unc.edu.pe/handle/UNC/1205

9. Instituto Nacional de Estadística e Informática (INEI). Estadísticas, noticias sobre anemia [Internet] [consultado 2020 Jun 3] Disponible en: https://www.inei.gob.pe/prensa/noticias/ la-sierra-presenta-los-mayores-niveles-de-anemia-del-pais-enel-ano-12223/

10. Lazarte A. Factores relacionados a la no adherencia del consumo de multimicronutrientes chispitas en madres de niños de 6 a 36 meses. Desafíos. 2018; 9(2), 23-33. Disponible en: https://doi.org/https://doi.org/10.37711/desafios.2018.9.2.131

11. Instituto Nacional de Estadística e Informática. Indicadores de Resultados de los Programas Presupuestales, Primer Semestre 2017 Encuesta Demográfica y de Salud Familiar (Resultados Preliminares) [Internet] [Consultado 2018 Ago 16] Disponible en: https://proyectos.inei.gob.pe/endes/images/ Indicadores_Resultados_PPR_Primer_Semestre_2017.pdf

12. Tinoco A, Yanzapanta K, Miranda K. Adherencia de la suplementación con multimicronutrientes y nivel de hemoglobina en niños de 6 a 36 meses del distrito de Huanca Huanca, Perú, 2017. RCCS [Internet] 2018 Dic 3;11(2): 40-47 [Consultado 2021 Mar 24] Disponible en: https://revistas.upeu. edu.pe/index.php/rc_salud/article/view/1106

13. Ortega J, Sánchez D, Rodríguez O, Ortega J. Adherencia terapéutica: un problema de atención médica. Acta méd. Grupo Ángeles [Internet] $2018 \mathrm{Sep}$; 16(3): 226-232 [consultado 2021 Feb 12] Disponible en: http://www.scielo.org.mx/scielo. php?script=sci_arttext\&pid=S187072032018000300226\&lng= es

14. Ramos E. La adherencia al tratamiento en las enfermedades crónicas. Rev cubana Angiol Cir Vasc [Internet] 2015 Dic; 16 (2): 175-189 [consultado 2021 Feb 12] Disponible en: http:// scielo.sld.cu/scielo.php?script=sci_arttext\&pid=S16820037201 $5000200006 \&$ Ing $=$ es

15. Laura G. Adherencia a la suplementación con sulfato ferroso en gotas de madres con lactantes de 4 meses, Centro De Salud I - 3 Coata, 2016 [Internet] Puno: Universidad Nacional del Altiplano; 2017. Disponible en: http://repositorio.unap.edu.pe/ handle/UNAP/5657 
16. Echagüe G, Funes P, Díaz V, Ruíz I, Ramírez M, Franco M, et al. Evaluación de anemia post intervención nutricional en niños de comunidades rurales de Caazapá, Paraguay. Pediatr. (Asunción) [Internet] 2019 Ago; 46(2): 103-109 [consultado 2021 Feb 12] Disponible en: http://scielo.iics.una.py/scielo. php?script=sci_arttext\&pid=\$168398032019000200103\&lng= en. https://doi.org/10.31698/ped.46022019006

17. Huamán L, Aparco P, Núñez E, Gonzáles E, Pillaca J, Mayta P. Consumo de suplementos con multimicronutrientes Chispitas y anemia en niños de 6 a 35 meses: estudio transversal en el contexto de una intervención poblacional en Apurímac, Perú. Rev. perú. med. exp. salud pública [Internet] 2012 Jul; 29(3): 314-323 [consultado 2021 Feb 12] Disponible en: http://www. scielo.org.pe/scielo.php?script=sci_arttext\&pid=S1726463420 $12000300004 \&$ Ing $=$ es

18. Aparco JP, Huamán-Espino L Barreras y facilitadores a la suplementación con micronutrientes en polvo: percepciones maternas y dinámica de los servicios de salud. Rev. perú. med. exp. salud pública [Internet] 2017 Oct; 34(4): 590-600[consultado 2021 Feb 16] Disponible en: http:// www.scielo.org.pe/scielo.php?script=sci_arttext\&pid=S172646342017000400003\&lng=es http://dx.doi.org/10.17843/ rpmesp.2017.344.3164

19. Inca $V$, Munares $O$. Factores asociados a la adherencia a suplementos de hierro en gestantes atendidas en un centro de salud de Cañete, Perú. Rev Int Salud Matern Fetal [Internet] 2020 Ene; 5(1) [consultado 2021 Feb 16] Disponible en: http://ojs.revistamaternofetal.com/index.php/RISMF/article/ view/149/176
20. Chávez M. Factores asociados a la falta de adherencia al consumo de Multimicronutrientes "Chispitas", en niños de 6 a 36 meses de edad, del Puesto De Salud I-2 Masusa, año 2018 [Internet] Iquitos: Universidad Nacional de la Amazonia Peruana; 2018 [consultado 2021 Feb 16] Disponible en: https:// alicia.concytec.gob.pe/vufind/Record/UNAP_05ad84f3b9420b c22b9ef4d4340be656

21. Vargas J. Actitudes de las madres de niños de 6 a 36 meses hacia la suplementación con multimicronutrientes en un establecimiento de salud de Lima, 2016 [Internet] Lima: Universidad nacional Mayor de San Marcos; 2017 [consultado 2021 Feb 16] Disponible en: https://cybertesis. unmsm.edu.pe/bitstream/handle/20.500.12672/5905/Vargas_ cj.pdf?sequence $=1$

22. Ipanaqué $P$, Taype $C$. Actitud de las madres de niños de 6 a 35 meses y su relación con el suministro de micronutrientes del centro de atención primaria de Breña, Lima-2018 [Internet] Lima: Universidad privada Norbert Wiener; 2019 [consultado 2020 Nov 12] Disponible en: http://repositorio.uwiener.edu.pe/ handle/123456789/2771

23. Apolinario R, Padilla $M$, Ramos R. Factores relacionados con las prácticas de las madres en la preparación y administración de micronutrientes en niños de 6 a 35 meses [Internet] Lima: Universidad Peruana Cayetano Heredia; 2018 [Consultado 2020 dic 22] Disponible en: http://repositorio.upch.edu.pe/ bitstream/handle/upch/3743/Factores_ApolinarioRojas_Cindy. pdf? sequence=1\&isAllowed=y. 